\title{
Discretizations for the Incompressible Navier-Stokes Equations Based on the Lattice Boltzmann Method
}

\author{
M. Junk $\quad$ A. Klar ${ }^{\dagger}$
}

May 19, 1999

\begin{abstract}
A discrete velocity model with spatial and velocity discretization based on a lattice Boltzmann method is considered in the low Mach number limit. A uniform numerical scheme for this model is investigated. In the limit, the scheme reduces to a finite difference scheme for the incompressible Navier-Stokes equation which is a projection method with a second order spatial discretization on a regular grid. The discretization is analyzed and the method is compared to Chorin's original spatial discretization. Numerical results supporting the analytical statements are presented.
\end{abstract}

Keywords. discrete velocity models, lattice Boltzmann method, low Mach number limit, incompressible Navier-Stokes equations, Chorin's projection scheme, finite difference method, relaxation systems

AMS subject classifications. $76 \mathrm{P} 05,76 \mathrm{D} 05,65 \mathrm{M} 06,35 \mathrm{~B} 25$

\section{$1 \quad$ Introduction}

Lattice Boltzmann methods (LBM) use discrete velocity models of kinetic equations to obtain approximate solutions of the incompressible Navier-Stokes system. The idea of LBM rests on the observation that the kinetic and the Navier-Stokes model are equivalent in the limit of small Knudsen and Mach numbers. See $[3,10]$ for reviews on lattice Boltzmann methods and [21] for a review on discrete velocity models. In recent years, numerous articles on LBM have appeared in which the

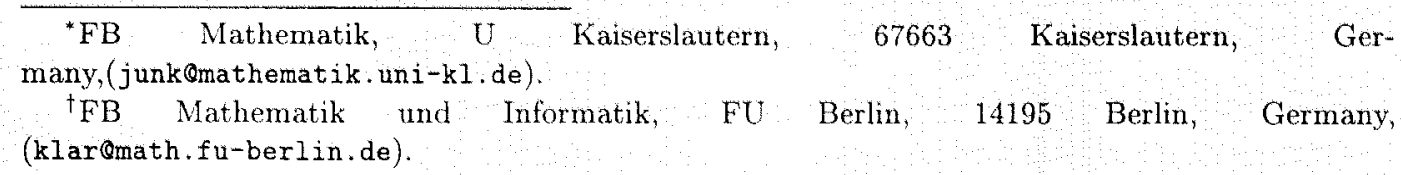


method is analyzed. We refer to the references in the above cited reviews and, e.g., to $[1,6,25]$. For connections to kinetic schemes, see [16].

A disadvantage of standard lattice Boltzmann discretizations is that the stiffness of the kinetic equation in the limit of small Knudsen and Mach numbers is not taken into account. Since the discretization is fully explicit, very fine time and space steps have to be used, slowing down the method considerably. To allow for larger discretization steps, the algorithm should at least be partially implicit. Such an approach has been successfully used for a large number of kinetic equations with stiff relaxation terms in fluid dynamic or diffusive limits and has led to the development of asymptotic preserving methods, see $[4,14,12,13,20,17,18]$. For a lattice Boltzmann type discrete velocity model with a diffusive scaling, a scheme working uniformly in the incompressible Navier Stokes limit, using a semi-implicit time discretization and leading to a Chorin projection scheme with MAC grid, has been suggested in [19].

In this work, our starting point is the following: numerically, it has been proven by many authors that the lattice Boltzmann method yields stable and reliable results for the incompressible Navier-Stokes equation [23, 11]. Moreover, pressure oscillations as in the original Chorin method, are not observed although the scheme works on a regular (collocated) grid. The aim, to extract the reason for this nice behavior, is accomplished by developing a method based on the LB spatial discretization and comparing it to Chorin's original method.

Our approach is based on the observation that the velocity-discrete kinetic equation is in one-to-one correspondence with a system of moment equations. The system includes the equations of mass and momentum which yield the Navier-Stokes equations in a suitable diffusion limit (related to small Knudsen and Mach numbers). Using essentially the space-discretization of LBM, we automatically obtain a discretization of the moment system which leads to a new spatial discretization for the incompressible Navier Stokes equations. The discretization is used together with the Chorin projection.

As has been investigated in detail for example in $[26,27]$, the original Chorin space discretization leads to an alternating error of first order in the pressure. This type of instability is not seen in the projection method if the MAC grid is used. In this case a second order approximation of the pressure is obtained without alternating terms in the error expansion for the pressure at first order. However, one has to use staggered grids having different locations for pressure and velocity. We mention that higher order approximation or regularizing methods have been used to avoid staggered grids and alternating terms in the error expansion (see [26]). It turns out that the spatial discretization of the scheme presented here is second order for both the pressure and the velocity, not using grid staggering. However, although the error in pressure is reduced compared to Chorin's scheme the errors in velocity are larger.

The paper is organized as follows: Section 2 introduces a lattice-Boltzmann type discrete velocity model and its associated closed moment system. In Section 3 the time 
and space discretization of the numerical scheme for the discrete velocity model is described. Section 4 deals with the low Mach number limit of the discretized equations leading to the projection method for the incompressible Navier-Stokes equations with the new spatial discretization. Section 5 contains remarks on the treatment of the boundary conditions and an analytical investigation of the scheme following the work in [26]. In particular, the scheme is compared to Chorin's original method. Finally, Section 6 presents a numerical investigation of the second order convergence for the pressure which has been found analytically. A numerical comparison with Chorin's scheme is included as well.

\section{A Lattice-Boltzmann type Discrete Velocity Model and the Associated Moment System}

The basic kinetic model is given by the Boltzmann equation

$$
\frac{\partial f}{\partial t}+\mathbf{v} \nabla f=J(f)
$$

which describes the evolution of a particle density $f(\mathbf{x}, \mathbf{v}, t)$. The left hand side of (1) represents free transport of the particles while the right hand side describes interactions through collisions. The difference between continuous and discrete velocity models is the structure of the phase space $\mathcal{X}^{\prime} \times \mathcal{V}$. In the classical Boltzmann equation, the space part $\mathcal{X}$ is a subset of $\mathbb{R}^{3}$ and the velocity domain $\mathcal{V}$ is the full space $\mathbb{R}^{3}$. For discrete models we have

$$
\mathcal{V}=\left\{\mathbf{c}_{0}, \ldots, \mathbf{c}_{N-1}\right\} \quad \mathbf{c}_{i} \in \mathbb{R}^{d} .
$$

In our particular example, we consider a two-dimensional model $(d=2)$ with nine velocities $(N=9)$

$$
\begin{array}{llll}
\mathbf{c}_{1}=\left(\begin{array}{l}
1 \\
0
\end{array}\right) & \mathbf{c}_{2}=\left(\begin{array}{l}
0 \\
1
\end{array}\right) & \mathbf{c}_{3}=\left(\begin{array}{c}
-1 \\
0
\end{array}\right) & \mathbf{c}_{4}=\left(\begin{array}{c}
0 \\
-1
\end{array}\right) \\
\mathbf{c}_{5}=\left(\begin{array}{l}
1 \\
1
\end{array}\right) & \mathbf{c}_{6}=\left(\begin{array}{c}
-1 \\
1
\end{array}\right) & \mathbf{c}_{7}=\left(\begin{array}{c}
-1 \\
-1
\end{array}\right) & \mathbf{c}_{8}=\left(\begin{array}{c}
1 \\
-1
\end{array}\right)
\end{array}
$$

and $\mathbf{c}_{0}=\mathbf{0}$. In the discrete case, the $\mathbf{v}$-dependence of the particle distribution $f(\mathbf{x}, \mathbf{v}, t)$ is uniquely determined through $N$ functions

$$
f_{i}(\mathbf{x}, t)=f\left(\mathbf{x}, \mathbf{c}_{i}, t\right) \quad i=0, \ldots, N-1
$$

which are called occupation numbers. Macroscopic quantities like mass-, momentumor energy density are obtained by taking velocity moments of $f$. If $\psi$ is any $\mathbf{v -}$ dependent function, we denote the discrete velocity integral by

$$
\langle\psi\rangle=\sum_{i=0}^{N-1} \psi\left(\mathbf{c}_{i}\right)
$$


Then, mass and momentum density can be written as

$$
\begin{array}{r}
\rho(\mathbf{x}, t)=\langle f(\mathbf{x}, \mathbf{v}, t)\rangle=\sum_{i=0}^{N-1} f_{i}(\mathbf{x}, t) \\
\rho \mathbf{u}(\mathbf{x}, t)=\langle\mathbf{v} f(\mathbf{x}, \mathbf{v}, t)\rangle=\sum_{i=0}^{N-1} \mathbf{c}_{i} f_{i}(\mathbf{x}, t)
\end{array}
$$

In lattice Boltzmann applications, the collision operator $J(f)$ in (1) is typically of BGK-type

$$
J(f)=-\frac{1}{\tau}\left(f-f^{e q}\right)
$$

The parameter $\tau>0$ is called relaxation time and $f^{e q}$ is the equilibrium distribution. In the isothermal case, $f^{e q}$ depends on $f$ through the parameters $\rho$ and $\mathbf{u}$ which are calculated according to (2). For the standard D2Q9-model [22] with 9 velocities, we have

$$
f^{e q}(\rho, \mathbf{u} ; \mathbf{v})=\rho\left(1+3 \mathbf{u} \cdot \mathbf{v}-\frac{3}{2}|\mathbf{u}|^{2}+\frac{9}{2}(\mathbf{u} \cdot \mathbf{v})^{2}\right) f^{*}(\mathbf{v})
$$

where $f^{*}$ is defined by

$$
f^{*}\left(\mathbf{c}_{i}\right)= \begin{cases}\frac{4}{9} & i=0 \\ \frac{1}{9} & i=1, \cdots, 4 \\ \frac{1}{36} & i=5, \cdots, 8\end{cases}
$$

The equilibrium distribution is constructed in such a way that

$$
\langle J(f)\rangle=0 \quad \text { and } \quad\langle\mathbf{v} J(f)\rangle=\mathbf{0}
$$

which reflects conservation of mass and momentum in the collision process.

In order to obtain a relation between the kinetic equation (1) and the incompressible Navier-Stokes system, we introduce the diffusive scaling $\mathbf{x} \rightarrow \mathbf{x} / \epsilon, t \rightarrow t / \epsilon^{2}$ together with a rescaling of velocity $\mathbf{u} \rightarrow \epsilon \mathbf{u}$ (see $[24,8,2]$ ). Under these transformations, (1) turns into

$$
\frac{\partial f}{\partial t}+\frac{1}{\epsilon} \mathcal{D} f=-\frac{1}{\epsilon^{2} \tau}\left(f-f^{e q}(\rho, \epsilon \mathbf{u})\right)
$$

where $\mathcal{D}=\mathbf{v} \cdot \nabla$ has been used as abbreviation for the space derivatives. In our exemplary case, (4) consists of nine equations for the occupation numbers $f_{0}, \ldots, f_{8}$. In order to get closer in notation to the Navier-Stokes system, we transform (4) into 
an equivalent set of moment equations (see also $[19,9]$ for a similar approach). Based on the v-polynomials

$$
\begin{aligned}
& P_{0}(\mathbf{v})=1 \\
& P_{1}(\mathbf{v})=\frac{v_{1}}{\epsilon} \\
& P_{3}(\mathbf{v})=\frac{v_{1}^{2}}{\epsilon^{2}}-\frac{1}{3 \epsilon^{2}} \\
& P_{2}(\mathbf{v})=\frac{v_{2}}{\epsilon} \\
& P_{6}(\mathbf{v})=\frac{\left(3|\mathbf{v}|^{2}-4\right) v_{1}}{\epsilon^{3}} \\
& P_{4}(\mathbf{v})=\frac{v_{1} v_{2}}{\epsilon^{2}} \\
& P_{5}(\mathbf{v})=\frac{v_{2}^{2}}{\epsilon^{2}}-\frac{1}{3 \epsilon^{2}} \\
& P_{8}(\mathbf{v})=\frac{9|\mathbf{v}|^{4}-15|\mathbf{v}|^{2}+2}{\epsilon^{4}}
\end{aligned}
$$

we obtain an invertible linear mapping $f \rightarrow \mathbf{P} f$ defined by

$$
\mathbf{P} f=\left(\left\langle P_{0} f\right\rangle, \ldots,\left\langle P_{8} f\right\rangle\right)^{T} .
$$

Applying $\mathbf{P}$ to equation (4) results in an equivalent set of equations with a differential operator which is still linear and hyperbolic

$$
\left(\frac{\partial}{\partial t}+\frac{1}{\epsilon} \mathbf{P} \mathcal{D} \mathbf{P}-1\right) \mathbf{P} f=-\frac{1}{\epsilon^{2} \tau}\left(\mathbf{P} f-\mathbf{P} f^{e q}(\rho, \epsilon \mathbf{u})\right)
$$

In order to write (6) in a more explicit form, we introduce names for the moments. Note that $\left\langle P_{0} f\right\rangle=\rho$ and $\left\langle P_{i} f\right\rangle=\rho u_{i}$ for $i=1,2$. The second order moments form a symmetric tensor

$$
\Theta=\left(\begin{array}{ll}
\Theta_{11} & \Theta_{12} \\
\Theta_{12} & \Theta_{22}
\end{array}\right)=\left(\begin{array}{ll}
\left\langle P_{3} f\right\rangle & \left\langle P_{4} f\right\rangle \\
\left\langle P_{4} f\right\rangle & \left\langle P_{5} f\right\rangle
\end{array}\right)
$$

and for the remaining moments we set

$$
\mathbf{q}=\left(\begin{array}{l}
\left\langle P_{6} f\right\rangle \\
\left\langle P_{7} f\right\rangle
\end{array}\right), \quad s=\left\langle P_{8} f\right\rangle .
$$

The first two equations in (6) are those of mass and momentum conservation

$$
\begin{gathered}
\partial_{t} \rho+\operatorname{div} \rho \mathbf{u}=0 \\
\partial_{t} \rho \mathbf{u}+\operatorname{div} \Theta+\frac{1}{3 \epsilon^{2}} \nabla \rho=0 .
\end{gathered}
$$

Here, the divergence is applied to the rows of $\Theta$. The equation for $\Theta$ is

$$
\partial_{t} \Theta+\frac{2}{3 \epsilon^{2}} S[\rho \mathbf{u}]+\frac{1}{3} Q=-\frac{1}{\epsilon^{2} \tau}(\Theta-\rho \mathbf{u} \otimes \mathbf{u})
$$

where

$$
S_{i j}[\rho \mathbf{u}]=\frac{1}{2}\left(\frac{\partial \rho u_{i}}{\partial x_{j}}+\frac{\partial \rho u_{j}}{\partial x_{i}}\right) \quad \text { and } \quad Q=\left(\begin{array}{cc}
\partial_{x_{2}} q_{2} & \partial_{x_{2}} q_{1}+\partial_{x_{1}} q_{2} \\
\partial_{x_{2}} q_{1}+\partial_{x_{1}} q_{2} & \partial_{x_{1}} q_{1}
\end{array}\right)
$$


Finally, the third and fourth order moments satisfy

$$
\begin{gathered}
\partial_{t} \mathbf{q}+\frac{1}{\epsilon^{2}} \operatorname{div}\left(\begin{array}{cc}
\Theta_{22} & 2 \Theta_{12} \\
2 \Theta_{12} & \Theta_{11}
\end{array}\right)+\frac{1}{6} \nabla s=-\frac{1}{\epsilon^{2} \tau} \mathbf{q} \\
\partial_{t} s+\frac{4}{\epsilon^{2}} \operatorname{div} \mathbf{q}=-\frac{1}{\epsilon^{2} \tau} s
\end{gathered}
$$

Altogether, we obtain a hyperbolic system with stiff relaxation terms. The diffusion limit of the above system is straightforwardly determined. From the momentum equation in (7) we conclude that $\nabla \rho$ tends to zero as $\epsilon \rightarrow 0$. Hence, $\rho$ approaches a constant $\ddot{\rho}$ (which is the Boussinesq relation in the isothermal case). Writing $\rho=\bar{\rho}\left(1+3 \epsilon^{2} p\right)$, equation (7) transforms into

$$
\begin{gathered}
\partial_{t} p{ }_{3 \epsilon^{2}}^{1} \operatorname{div} \mathbf{u}=-\operatorname{div} p \mathbf{u} \\
\partial_{t} \mathbf{u}+\operatorname{div} \frac{1}{\bar{\rho}} \Theta+\nabla p=-3 \epsilon^{2} \partial_{t} p \mathbf{u}
\end{gathered}
$$

For $\epsilon \rightarrow 0$, equation (8) yields in lowest order

$$
\frac{1}{\bar{\rho}} \Theta=\mathbf{u} \otimes \mathbf{u}-\frac{2 \tau}{3} S[\mathbf{u}]
$$

Since (9) decouples completely from the other equations (in lowest order) and since $2 \operatorname{div} S[\mathbf{u}]=(\Delta+\nabla \operatorname{div}) \mathbf{u}$, we obtain from (10) and (11) the incompressible NavierStokes equations as limiting system

$$
\begin{gathered}
\operatorname{div} \mathbf{u}=0 \\
\partial_{t} \mathbf{u}+\operatorname{div} \mathbf{u} \otimes \mathbf{u}+\nabla p=\frac{\tau}{3} \Delta \mathbf{u}
\end{gathered}
$$

The Reynolds number is related to the relaxation time by $R e=3 / \tau$.

We remark that (7), (8) and (9) can be viewed as a relaxation system for (12). Since LBM is a particular discretization of (4) and since a discretization of (4) automatically turns into a discretization of the moment system under the transformation $\mathbf{P}$, we conclude that LBM can be viewed as a relaxation method for the incompressible Navier-Stokes system [16].

\section{The Numerical Scheme: Spatial and temporal dis- cretization}

To obtain a spatial discretization of the moment system $(7),(8)$ and $(9)$, we use a first order upwind method for the operator $\mathcal{D}=\mathrm{v} \cdot \nabla$ in (6). This choice is motivated by the lattice Boltzmann method where the upwind approximation is 
combined with an explicit Euler discretization for the time derivative. To get a proper treatment of the stiff pressure-velocity coupling, however, we choose a semiimplicit time discretization instead of the explicit one (see also [19]). In the limit $\epsilon \rightarrow$ 0 , a projection scheme for the incompressible Navier Stokes equations is obtained.

Before we describe the discretization of the moment system, let us first show how the lattice Boltzmann method is obtained in this context, see also [5]. The discretization of $\mathcal{D}=\mathbf{v} \cdot \nabla$ is taken as

$$
\left(\mathcal{D}_{h} f\right)(\mathbf{x}, \mathbf{v}, t)-\frac{1}{h}(f(\mathbf{x}, \mathbf{v}, t)-f(\mathbf{x}-h \mathbf{v}, \mathbf{v}, t))
$$

Together with an explicit Euler discretization of the time derivative and an evaluation of the collision operator at a shifted $\mathrm{x}$-position, (4) turns into

$$
\begin{aligned}
f(\mathbf{x}, \mathbf{v}, t+\Delta t)-f(\mathbf{x}, \mathbf{v}, t)+\frac{\Delta t}{\epsilon h}(f(\mathbf{x}, \mathbf{v}, t) & -f(\mathbf{x}-h \mathbf{v}, \mathbf{v}, t)) \\
& =-\frac{\Delta t}{\epsilon^{2} \tau}\left(f-f^{e q}(\rho, \epsilon \mathbf{u})\right)(\mathbf{x}-h \mathbf{v}, \mathbf{v}, t)
\end{aligned}
$$

Setting $\Delta t=\epsilon^{2}, h=\epsilon, \mathbf{v}=t \mathbf{w}$ and transforming the space variable $\mathbf{x} \rightarrow \mathbf{x}+\mathbf{w} \Delta l$ yields for the occupation numbers

$$
f_{i}\left(\mathbf{x}+\mathbf{w}_{i} \Delta t, t+\Delta t\right)-f_{i}(\mathbf{x}, t)=-\frac{1}{\tau}\left(f_{i}(\mathbf{x}, t)-f_{i}^{e q}(\rho(\mathbf{x}, t), \epsilon \mathbf{u}(\mathbf{x}, t))\right)
$$

which is the standard discretization in LBM (sce $[22,6,1])$.

To describe the effect of the discretization (13) on the moment system, we introduce the vectors

$$
\mathbf{M}=\mathbf{P} f=\left(\rho, \rho u_{1}, \rho u_{2}, \Theta_{11}, \Theta_{12}, \Theta_{22}, q_{1}, q_{2}, s\right)^{T}
$$

and

$$
\mathbf{M}^{e q}=\mathbf{P} f^{e q}(\rho, \epsilon \mathbf{u})=\left(\rho, \rho u_{1}, \rho u_{2}, \rho u_{1}^{2}, \rho u_{1} u_{2}, \rho u_{2}^{2}, 0,0,0\right)^{T} .
$$

Using (13) to replace $\mathcal{D}$ in (6), the discretized moment system can be written in the compact form

$$
\frac{\partial \mathbf{M}}{\partial t}+\frac{1}{\epsilon} \mathbf{P} \mathcal{D}_{h} \mathbf{P}^{-1} \mathbf{M}=-\frac{1}{\tau \epsilon^{2}}\left(\mathbf{M}-\mathbf{M}^{e q}\right)
$$

If $\left\{\mathbf{e}_{i}: i-0, \ldots, 8\right\}$ are the standard unit vectors in $\mathbb{R}^{9}$, we can write the contribution of $M_{j}$ to the equation for $M_{i}$ as

$$
\frac{1}{\epsilon} \mathbf{e}_{i} \cdot\left(\mathbf{P} \mathcal{D}_{h} \mathbf{P}^{-1}\right) M_{j} \mathbf{e}_{j}=\frac{1}{\epsilon} \mathbf{e}_{i} \cdot\left(\mathbf{P} \frac{1}{h}\left(M_{j}(\mathbf{x}, t)-M_{j}(\mathbf{x}-h \mathbf{v})\right) \mathbf{P}^{-1} \mathbf{e}_{j}\right)
$$


With the abbreviation $g_{j}=\mathbf{P}^{-1} \mathbf{e}_{j}$, (14) can be transformed into

$$
\begin{aligned}
\frac{1}{\epsilon} \mathbf{e}_{i} \cdot\left(\mathbf{P} \mathcal{D}_{h} \mathbf{P}^{-1}\right) M_{j} \mathbf{e}_{j} & =\frac{1}{\epsilon h}\left(M_{j}(\mathbf{x}, t) \delta_{i j}-\left\langle P_{i}(\mathbf{v}) g_{j}(\mathbf{v}) M_{j}(\mathbf{x}-\mathbf{v} h)\right\rangle\right) \\
& =\frac{1}{\epsilon h}\left(M_{j}(\mathbf{x}, t) \delta_{i j}-\sum_{k=0}^{8} P_{i}\left(\mathbf{c}_{k}\right) g_{j}\left(\mathbf{c}_{k}\right) M_{j}\left(\mathbf{x}-\mathbf{c}_{k} h\right)\right)
\end{aligned}
$$

Hence, the contribution of $M_{j}$ to equation $i$ can be written as a finite difference stencil with the general form

$$
\frac{1}{\epsilon h}\left[\begin{array}{ccc}
-P_{i} g_{j}\left(\mathbf{c}_{8}\right) & -P_{i} g_{j}\left(\mathbf{c}_{4}\right) & -P_{i} g_{j}\left(\mathbf{c}_{7}\right) \\
-P_{i} g_{j}\left(\mathbf{c}_{1}\right) & \delta_{i j}-P_{i} g_{j}\left(\mathbf{c}_{0}\right) & -P_{i} g_{j}\left(\mathbf{c}_{3}\right) \\
-P_{i} g_{j}\left(\mathbf{c}_{5}\right) & -P_{i} g_{j}\left(\mathbf{c}_{2}\right) & -P_{i} g_{j}\left(\mathbf{c}_{6}\right)
\end{array}\right] .
$$

In particular, for each space derivative in the moment system (7), (8) and (9) a corresponding stencil is found. The discretization of $x_{1}$ and $x_{2}$-derivatives occurs in three different ways. For div $\rho \mathbf{u}$ in the mass conservation equation as well as for $\nabla \rho$ in the momentum equation the following stencils appear

$$
\frac{\partial}{\partial x_{1}} \approx \frac{1}{12 h}\left[\begin{array}{ccc}
-1 & 0 & 1 \\
-4 & 0 & 4 \\
-1 & 0 & 1
\end{array}\right], \quad \frac{\partial}{\partial x_{2}} \approx \frac{1}{12 h}\left[\begin{array}{ccc}
1 & 4 & 1 \\
0 & 0 & 0 \\
-1 & -4 & -1
\end{array}\right]
$$

They also occur in the momentum equation to approximate $\partial_{x_{1}} \Theta_{11}, \partial_{x_{2}} \Theta_{22}$ and in equation (8) for $\partial_{x_{1}} \rho u_{1}, \partial_{x_{2}} \rho u_{2}$. The derivatives of $\Theta_{12}$ as well as $\rho u_{1} u_{2}$ are always discretized by

$$
\frac{\partial}{\partial x_{1}} \approx \frac{1}{4 h}\left[\begin{array}{ccc}
-1 & 0 & 1 \\
0 & 0 & 0 \\
-1 & 0 & 1
\end{array}\right], \quad \frac{\partial}{\partial x_{2}} \approx \frac{1}{4 h}\left[\begin{array}{ccc}
1 & 0 & 1 \\
0 & 0 & 0 \\
-1 & 0 & -1
\end{array}\right]
$$

These stencils also appear in (8) for $\partial_{x_{1}} \rho u_{2}, \partial_{x_{2}} \rho u_{2}$ and $\partial_{x_{1}} q_{2}, \partial_{x_{2}} q_{1}$. The remaining derivatives in the moment system turn out to be approximated by

$$
\frac{\partial}{\partial x_{1}} \approx \frac{1}{6 h}\left[\begin{array}{lll}
-1 & 0 & 1 \\
-1 & 0 & 1 \\
-1 & 0 & 1
\end{array}\right], \quad \frac{\partial}{\partial x_{2}} \approx \frac{1}{6 h}\left[\begin{array}{ccc}
1 & 1 & 1 \\
0 & 0 & 0 \\
-1 & -1 & -1
\end{array}\right]
$$

We remark that each stencil is second order accurate. In fact, the stencils can be viewed as convex combinations of standard central difference approximations. For example, the $\partial_{x_{1}}$-stencil in (16) can be written as

$$
\frac{1}{12 h}\left[\begin{array}{lll}
-1 & 0 & 1 \\
-4 & 0 & 4 \\
-1 & 0 & 1
\end{array}\right]=\frac{1}{6}\left(\frac{1}{2 h}\left[\begin{array}{ccc}
-1 & 0 & 1 \\
0 & 0 & 0 \\
0 & 0 & 0
\end{array}\right]+\frac{4}{2 h}\left[\begin{array}{ccc}
0 & 0 & 0 \\
-1 & 0 & 1 \\
0 & 0 & 0
\end{array}\right]+\frac{1}{2 h}\left[\begin{array}{ccc}
0 & 0 & 0 \\
0 & 0 & 0 \\
-1 & 0 & 1
\end{array}\right]\right)
$$


Up to now, we have investigated the approximations of the space derivatives appearing in the moment system. However, if we consider, for example, the discretized mass conservation equation, we find that apart from $M_{1}=\rho u_{1}$ and $M_{2}=\rho u_{2}$ also the other variables $M_{0}, M_{3}, \ldots, M_{8}$ contribute. Of course, this contribution is of higher order in the discretization parameter $h$ and the appearance of such terms is not surprising since we started with a first order upwind approximation (13). As example, we mention the contribution of $\rho$ to the mass conservation equation (i.e. $i=j=0$ in (15)). Evaluating (15), we find up to a constant factor

$$
\frac{1}{6 h}\left[\begin{array}{lll}
-1 & -4 & -1 \\
-4 & 20 & -4 \\
-1 & -4 & -1
\end{array}\right]=-h \Delta+\mathcal{O}\left(h^{3}\right)
$$

which is the standard nine-point stencil for the Laplacian. If we keep all terms which appear in that way and which are of first order in $h$, we obtain the modified equation of the finite difference approximation (i.e. the equation which is approximated to second order accuracy). The modified equation corresponding to (7) is

$$
\begin{gathered}
\partial_{t} \rho+\operatorname{div} \rho \mathbf{u}=\left(\frac{1}{6 \epsilon} \Delta \rho+\frac{\epsilon}{2} \operatorname{div} \operatorname{div} \Theta\right) h \\
\partial_{t} \rho \mathbf{u}+\operatorname{div} \Theta+\frac{1}{3 \epsilon^{2}} \nabla \rho=\left(\frac{1}{6 \epsilon}(\Delta+2 \nabla \operatorname{div}) \rho \mathbf{u}+\frac{\epsilon}{6} \operatorname{div} Q\right) h
\end{gathered}
$$

Similarly, equations are obtained for $\Theta, \mathbf{q}$ and $s$. Note that the additional terms have a stabilizing effect because they act as artificial viscosity. In the original lattice Boltzmann approach, this viscosity is partly compensated by a negative viscosity due to the explicit Euler discretization of the time derivative and partly it is combined with the physical viscosity in the Navier-Stokes equation (which is possible since $(\Delta+2 \nabla$ div $) \rho \mathbf{u}$ has the correct structure). In this way, second order accuracy of LBM is obtained. Since we want to avoid the explicit time discretization but still obtain a second order accurate scheme, we neglect all stencils (15) which do not have counterparts in the moment system. With this step, we focus only on the interplay between the stencils (16), (17) and (18). Another possibility is to start with a second order upwind discretization of $\mathcal{D}$ instead of the first order approach (13) (compare here for example [14]).

To introduce the time discretization, we will not replace the space derivatives by their finite difference approximations $(16),(17)$ and (18) but restrict to the spatially continuous case to avoid confusion. Of course, the complete scheme is obtained by combining both space and time discretizations.

Introducing the momentum vector $\mathbf{m}^{k}(\mathbf{x})=\rho \mathbf{u}(\mathbf{x}, k \Delta t)$ and the pressure variable $p^{k}$ by $\rho(\mathbf{x}, k \Delta t)=\ddot{\rho}\left(1+3 \epsilon^{2} p^{k}(\mathbf{x})\right)$, we define $p^{k+1}$ and $\mathbf{m}^{k+1}$ based on a semi-implicit discretization of $(7)$

$$
\begin{gathered}
p^{k+1}=p^{k}-\frac{\Delta t}{3 \widetilde{\rho} \epsilon^{2}} \operatorname{div} \mathbf{m}^{k+1} \\
\mathbf{m}^{k+1}=\mathbf{m}^{k}-\Delta t\left(\operatorname{div} \Theta^{k}+\bar{\rho} \nabla p^{k+1}\right)
\end{gathered}
$$


Inserting $\mathbf{m}^{k+1}$ into the pressure equation yields a Helmholtz problem

$$
\left(\Delta-\frac{3 \epsilon^{2}}{\Delta t^{2}}\right) p^{k+1}=\frac{1}{\bar{\rho} \Delta t} \operatorname{div} \mathbf{m}^{k}-\operatorname{div} \operatorname{div} \frac{1}{\bar{\rho}} \Theta^{k}-\frac{3 \epsilon^{2}}{\Delta t^{2}} p^{k}
$$

We remark that the discretization of the second order operators $\Delta$ and div div is automatically given by a composition of the discretizations (16), (17) and (18). As time discretization of $(8)$, we choose

$$
\Theta^{k+1}=\Theta^{k}-\frac{2 \Delta t}{3 \epsilon^{2}} S\left[\mathbf{m}^{k+1}\right]+\frac{\Delta t}{3} Q^{k}-\frac{\Delta t}{\epsilon^{2} \tau}\left(\Theta^{k+1}-\left(\frac{\mathbf{m} \otimes \mathbf{m}}{\rho}\right)^{k+1}\right)
$$

Finally, equation (9) is treated according to

$$
\begin{gathered}
\mathbf{q}^{k+1}=\mathbf{q}^{k}-\frac{\Delta t}{\epsilon^{2}} \operatorname{div}\left(\begin{array}{cc}
\Theta_{22} & 2 \Theta_{12} \\
2 \Theta_{12} & \Theta_{11}
\end{array}\right)^{k}-\frac{\Delta t}{6} \nabla s^{k+1}-\frac{\Delta t}{\epsilon^{2} \tau} \mathbf{q}^{k+1} \\
s^{k+1}=s^{k}-\frac{4 \Delta t}{\epsilon^{2}} \operatorname{div} \mathbf{q}^{k+1}-\frac{\Delta t}{\epsilon^{2} \tau} s^{k+1}
\end{gathered}
$$

which again leads to an elliptic problem (with $\alpha=\tau / \Delta t$ )

$$
\begin{aligned}
\left(1-\frac{4 \tau^{2} \epsilon^{2}}{6\left(1+\epsilon^{2} \alpha\right)^{2}} \Delta\right) s^{k+1}=\frac{\epsilon^{2} \alpha}{1+\epsilon^{2} \alpha} s^{k} & -\frac{4 \tau \epsilon^{2} \alpha}{\left(1+\epsilon^{2} \alpha\right)^{2}} \operatorname{div} \mathbf{q}^{k} \\
& +\frac{4 \tau^{2}}{\left(1+\epsilon^{2} \alpha\right)^{2}} \operatorname{div} \operatorname{div}\left(\begin{array}{cc}
\Theta_{22} & 2 \Theta_{12} \\
2 \Theta_{12} & \Theta_{11}
\end{array}\right)^{k}
\end{aligned}
$$

The elliptic problems for $p$ and $s$, which result from the semi-implicit treatment, can be solved by an iterative procedure. The remaining equations for $\mathbf{m}, \Theta$ and $\mathbf{q}$ are explicit.

\section{The discretization of the incompressible Navier-Stokes equations}

The discretization of the moment system described in the last section tends, as $\epsilon \rightarrow 0$, to a Chorin type projection method for the incompressible Navier-Stokes equations. Since $\rho \rightarrow \bar{\rho}$ in that limit, we have $\mathbf{m}=\rho \mathbf{u} \rightarrow \bar{\rho} \mathbf{u}$ and the momentum equation in (20) yields

$$
\mathbf{u}^{k+1}=\mathbf{u}^{k}-\Delta t \operatorname{div} \frac{1}{\bar{\rho}} \Theta^{k}-\Delta t \nabla p^{k+1}
$$

The Helmholtz equation (21) for the pressure turns into

$$
\Delta p^{k+1}=\frac{1}{\Delta t} \operatorname{div} \mathbf{u}^{k}-\operatorname{div} \operatorname{div} \frac{1}{\bar{\rho}} \Theta^{k}
$$


Both (23) and (24) are combined with the limit of (22)

$$
\frac{1}{\bar{\rho}} \Theta^{k+1}=\mathbf{u}^{k+1} \otimes \mathbf{u}^{k+1}-\frac{2 \tau}{3} S\left[\mathbf{u}^{k+1}\right]
$$

For (23), this implies

$$
\mathbf{u}^{k+1}=\mathbf{u}^{k}-\Delta t \operatorname{div} \mathbf{u}^{k} \otimes \mathbf{u}^{k}-\Delta t \nabla p^{k+1}+\frac{2 \tau \Delta t}{3} \operatorname{div} S\left[\mathbf{u}^{k}\right]
$$

By construction, each differential operator in (26) is composed of derivatives from the original moment system for which we have derived the discretizations $(16),(17)$ and (18). If $\bar{D}_{1}, \bar{D}_{2}$ denote the approximations in (16) and $\tilde{D}_{1}, \tilde{D}_{2}$ those in (17), we find for the pressure gradient and the convective term in (26)

$$
\nabla p \leftrightarrow \bar{G} p=\left(\begin{array}{c}
\bar{D}_{1} p \\
\bar{D}_{2} p
\end{array}\right), \quad \operatorname{div} \mathbf{u} \otimes \mathbf{u} \leftrightarrow \hat{D} \mathbf{u}^{k} \otimes \mathbf{u}^{k}=\left(\begin{array}{l}
\bar{D}_{1} u_{1}^{2}+\tilde{D}_{2} u_{1} u_{2} \\
\tilde{D}_{1} u_{1} u_{2}+\bar{D}_{2} u_{2}^{2}
\end{array}\right) .
$$

The viscous term is of the form

$$
2 \operatorname{div} S[\mathbf{u}] \leftrightarrow \hat{L} \mathbf{u}=\left(\begin{array}{l}
2 \tilde{D}_{1}^{2} u_{1}+\tilde{D}_{2}^{2} u_{1}+\tilde{D}_{2} \tilde{D}_{1} u_{2} \\
\tilde{D}_{1} \tilde{D}_{2} u_{1}+\tilde{D}_{1}^{2} u_{2}+2 \tilde{D}_{2}^{2} u_{2}
\end{array}\right)
$$

Under discrete divergence-free condition, $\bar{D} \mathbf{u}=0$, a reorganization yields

$$
\left(\begin{array}{l}
2 \tilde{D}_{1}^{2} u_{1}+\tilde{D}_{2}^{2} u_{1}+\tilde{D}_{2} \tilde{D}_{1} u_{2} \\
\tilde{D}_{1} \tilde{D}_{2} u_{1}+\tilde{D}_{1}^{2} u_{2}+2 \bar{D}_{2}^{2} u_{2}
\end{array}\right)=\left(\begin{array}{l}
\bar{D}_{1}^{2} u_{1}+\tilde{D}_{2}^{2} u_{1} \\
\tilde{D}_{1}^{2} u_{2}+\bar{D}_{2}^{2} u_{2}
\end{array}\right)+\left[\tilde{D}_{1} \tilde{D}_{2}, \bar{D}_{1} \bar{D}_{2}\right] \mathbf{u}
$$

where $[\cdot$,$] denotes the commutator. Since both \tilde{D}_{1} \tilde{D}_{2}$ and $\bar{D}_{1} \bar{D}_{2}$ are second order accurate approximations of the mixed derivative $\partial_{x_{1}} \partial_{x_{2}}$, the commutator vanishes in the order of accuracy of the method

$$
\left[\tilde{D}_{1} \tilde{D}_{2}, \bar{D}_{1} \tilde{D}_{2}\right] \mathbf{u}=\mathcal{O}\left(h^{2}\right)
$$

Thus, $\hat{L}$ is a second order approximation of the Laplacian. Finally, div $\mathbf{u}^{k}$ and the Laplacian in (24) turn out to be discretized by

$$
\begin{gathered}
\operatorname{div} \mathbf{u} \leftrightarrow \vec{D} \mathbf{u}=\breve{D}_{1} u_{1}+\bar{D}_{2} u_{2}, \\
\Delta p \leftrightarrow \bar{L} p=\bar{D} \bar{G} p=\left(\bar{D}_{1}^{2}+\bar{D}_{2}^{2}\right) p .
\end{gathered}
$$

According to (25), the double divergence in (24) splits into two contributions. The convective part div div $\mathbf{u} \otimes \mathbf{u}$ is discretized by $\bar{D} \hat{D} \mathbf{u} \otimes \mathbf{u}$ where $\hat{D} \mathbf{u} \otimes \mathbf{u}$ is given in (27). Similarly, the part involving third order derivatives $\bar{D} \hat{D} S[\mathbf{u}]$. Altogether, the discrete versions of (24) and (26) can be written as

$$
\widetilde{L} p^{k+1}=\frac{1}{\Delta t} \tilde{D} \mathbf{u}^{k}-\tilde{D} \hat{D} \mathbf{u}^{k} \otimes \mathbf{u}^{k}+\frac{\tau}{3} \tilde{D} \hat{L} \mathbf{u}^{k}
$$

and

$$
\mathbf{u}^{k+1}=\mathbf{u}^{k}-\Delta t \hat{D} \mathbf{u}^{k} \otimes \mathbf{u}^{k}-\Delta t \bar{G} p^{k+1}+\frac{\tau \Delta t}{3} \hat{L} \mathbf{u}^{k}
$$

which is a Chorin-type projection method with spatial discretization induced by the lattice Boltzmann approach. 


\section{Boundary conditions and remarks on alternating er- rors}

In this section appropriate boundary conditions for our method are introduced and oscillating pressure error terms are discussed. The following investigations are motivated by the detailed analysis of finite difference schemes for the incompressible Navier-Stokes equations in [26].

In Chorin's method [7], the pressure is only first order accurate (with respect to the spatial discretization parameter $h$ ) despite the fact that all finite difference approximations are second order consistent. As shown in [26], this behavior results from an interaction of the discretized viscous term in the Navier-Stokes equations and the pressure Poisson equation. More precisely, the wide stencil for the Laplacian in the pressure equation essentially decouples the problem into two Poisson problems on separate sub-grids. Since the sub-problems are subject to boundary conditions which differ at third order, alternating errors at third order are introduced (i.e., error terms are present on one sub-grid and absent on the other). If the Laplacian in the viscous term is discretized with the standard five-point stencil, as in Chorin's method, the application to an alternating function yields contributions of order $1 / h^{2}$. In this way, the third order oscillating error is brought down to first order. To avoid this phenomenon, several approaches have been proposed and some of them are analyzed in [26]. In the lattice Boltzmann induced discretization of the Navier-Stokes equations described in Section 4, the accuracy of the pressure remains second order. In contrast to the methods investigated in [26], an amplification of the alternating error at third order is avoided due to the wide stencil $\hat{L}$ for the Laplacian in the viscous term.

To explain the basic principles which lead to the alternating error terms in Chorin's method, we consider the Stokes equation in a periodic channel as a model problem. The extension of the boundary treatment to the new discretization will then be straight forward.

By neglecting the nonlinear terms in the Navier-Stokes equations, we obtain the Stokes equation

$$
\begin{gathered}
\partial_{t} \mathbf{u}+\nabla p=\nu \Delta \mathbf{u} \\
\operatorname{div} \mathbf{u}=0 \quad \text { in } \Omega,\left.\quad \mathbf{u}\right|_{t=0}=\mathbf{u}_{0}
\end{gathered}
$$

As in [26], we choose $\Omega=(0,1) \times(0,1)$ with no-slip conditions $\mathbf{u}=\mathbf{0}$ on the top and bottom boundaries and periodic conditions in horizontal direction. The pressure $p$ is determined by the constraint div $\mathbf{u}=0$, i.e. $p$ has to be chosen in such a way that the gradient part of the vector field

$$
\mathbf{a}-\nu \Delta \mathbf{u}
$$


is exactly compensated. Following the notation in [26], the divergence free projection of $\mathbf{a}$ is given by

$$
\mathcal{P a}=\mathbf{a}-\nabla q
$$

where $q$ solves the Neumann-Poisson problem

$$
\Delta q=\operatorname{div} \mathbf{a} \quad \text { in } \Omega, \quad \frac{\partial q}{\partial \mathbf{n}}=\mathbf{n} \cdot \mathbf{a} \text { on } \partial \Omega
$$

with a normalization condition $\int_{\Omega} q d x=0$. To get a discrete representation of the projection operator, we introduce discretizations $G$ and $D$ of the gradient and the divergence. Then, the discrete divergence-free part $\mathcal{P}_{h} \mathbf{a}$ of a is obtained by solving

$$
D G q=D \mathbf{a}
$$

with suitably discretized Neumann and normalization conditions. Indeed, sething

$$
\mathcal{P}_{h} \mathbf{a}-\mathbf{a}-G q
$$

we find, by construction, $D \mathcal{P}_{h} \mathbf{a}=0$.

In Chorin's method, $G$ and $D$ are based on standard central difference approximations on the regular square grid $\Omega_{h}=\left\{x_{i j}=(i h, j h): i, j=0, \ldots, N\right\}$ where $h=1 / N$. With

$$
D_{1}=\frac{1}{2 h}\left[\begin{array}{lll}
-1 & 0 & 1
\end{array}\right] \quad \text { and } \quad D_{2}=\frac{1}{2 h}\left[\begin{array}{c}
1 \\
0 \\
-1
\end{array}\right]
$$

the discrete gradient acting on a scalar function $q$ is given by

$$
(G q)_{i j}=\left(\begin{array}{l}
\left(D_{1} q\right)_{i j} \\
\left(D_{2} q\right)_{i j}
\end{array}\right)=\frac{1}{2 h}\left(\begin{array}{c}
q_{i+1, j}-q_{i-1, j} \\
q_{i, j+1}-q_{i, j-1}
\end{array}\right)
$$

(The weight in the center of the stencil always refers to the point where the stencil is applied.) At points $x_{i j}$ well inside the domain $(2 \leq j \leq N-2)$, the divergence $D$ acts on a vector field $u$ according to

$$
D \mathbf{u}=D_{1} u_{1}+D_{2} u_{2}
$$

The iterated operator $L=D G$ appearing in (30) is then the wide Laplacian

$$
L=\frac{1}{4 h^{2}}\left[\begin{array}{ccccc} 
& & 1 & & \\
& 0 & & \\
1 & 0 & -4 & 0 & 1 \\
& 0 & & \\
1 & &
\end{array}\right]
$$


At points $x_{i j}$ next to the boundary $(j=1$ or $j=N-1)$, the original operator $D_{2}$. is, according to Chorin's approach, replaced by

$$
D_{2}^{(1)}=\frac{1}{2 h}\left[\begin{array}{l}
1 \\
0 \\
0
\end{array}\right] \quad \text { and } \quad D_{2}^{(N-1)}=\frac{1}{2 h}\left[\begin{array}{c}
0 \\
0 \\
-1
\end{array}\right]
$$

For the velocity field $\mathbf{u}$, the discrete operator

$$
\left(D^{(j)} \mathbf{u}\right)_{i j}=\left(D_{1} u_{1}\right)_{i j}+\left(D_{2}^{(j)} u_{2}\right)_{i j} \quad j \in\{1, N-1\}
$$

still approximates the divergence since $\mathbf{u}=\mathbf{0}$ on the boundary so that $\left(D^{(j)} \mathbf{u}\right)_{i j}=$ $(D \mathbf{u})_{i j}$. However,

$$
L^{(1)}=D^{(1)} G=D_{1} D_{1}+D_{2}^{(1)} D_{2}=\frac{1}{4 h^{2}}\left[\begin{array}{cccc} 
& 1 \\
0 & & \\
1 & -3 & 0 & 1 \\
0 & 0 &
\end{array}\right]
$$

now has the interpretation as a discrete Laplacian with an incorporated boundary condition. Indeed, $\left(L^{(1)} q\right)_{i 1}=\left(D^{(1)} \mathbf{a}\right)_{i 1}$ can be rewritten in terms of the undisturbed operators $(L q)_{i 1}=(D \mathbf{a})_{i 1}$ together with the condition

$$
\left(D_{2} q\right)_{i 0}=\left(a_{2}\right)_{i 0}
$$

which is a second order accurate approximation of the Neumann boundary condition $\frac{\partial q}{\partial \mathbf{n}}=\mathbf{a} \cdot \mathbf{n}$ at the lower boundary. (Similar considerations apply to the upper boundary.)

Finally, in boundary points, the $x_{1}$-derivative of the divergence is deleted completely $\left(D_{1}^{(j)}=0\right.$ for $\left.j \in\{0, N\}\right)$ and the $x_{2}$-derivative is obtained from the half-sided difference

$$
D_{2, h s}=\frac{1}{2 h}\left[\begin{array}{c}
-1 \\
4 \\
-3 \\
0 \\
0
\end{array}\right]
$$

by deleting the central weight. The equation $\left(L^{(0)} q\right)_{i 0}=\left(D^{(0)} \mathbf{a}\right)_{i 0}$ based on the iterated operator

$$
L^{(0)}=D^{(0)} G=D_{1}^{(0)} D_{1}+D_{2}^{(0)} D_{2}
$$

can again be interpreted as $(L q)_{i 0}=(D \mathbf{a})_{i 0}$ but now with the boundary condition

$$
\left(B D_{2} q-B a_{2}\right)_{i, 0}=\frac{h}{3}\left(D_{1} D_{1} q-D_{1} a_{1}\right)_{i, 0} .
$$


Here, $B$ is the linear interpolation operator

$$
B=\frac{1}{3}\left[\begin{array}{c}
-1 \\
3 \\
0 \\
1 \\
0
\end{array}\right]=1+\mathcal{O}\left(h^{3}\right)
$$

so that $B\left(D_{2} q-a_{2}\right)$ also approximates the Neumann condition.

Before we apply the construction of the boundary stencils to the discretization induced by the lattice Boltzmann method, let us briefly comment on the mechanism which introduces alternating errors (see also [26]). An important observation is that the wide Laplacian (31) involves either even layers $(j=0,2, \ldots, N)$, or odd layers $(j=1,3, \ldots, N-1)$ but does not mix in between them (we assume for simplicity that $N$ is even). Since the boundary stencils $L^{(1)}$ and $L^{(N-1)}$ respect this separation, the Poisson problem on the odd sub-grid decouples completely. (Here, we assume for simplicity that the integral normalization is discretized separately on each sub-grid. Moreover, the accuracy of the integration rule is assumed to be at least fourth order which again simplifies the argument.) To analyze the solution of

$$
\begin{gathered}
\left(L q_{\text {odd }}-D \mathbf{a}\right)_{i j}=0 \quad j=1,3, \ldots, N-1 \\
\left(D_{2} q_{\text {odd }}-a_{2}\right)_{i j}=0 \quad j \in\{1, N-1\}
\end{gathered}
$$

with the discretized integral normalization, we assume an expansion of the form

$$
q_{\text {odd }}=q_{\text {odd }}^{(0)}+h q_{\text {odd }}^{(1)}+h^{2} q_{\text {odd }}^{(2)}+h^{3} q_{\text {odd }}^{(3)}+\mathcal{O}\left(h^{4}\right) .
$$

Inserting (35) into (34), performing a Taylor expansion and considering equal orders in $h$, we find equations satisfied by the coefficients. In lowest order, the original problem

$$
\Delta q^{(0)}=\operatorname{diva}, \quad \frac{\partial q^{(0)}}{\partial x_{2}}=a_{2} \quad \text { on } \partial \Omega, \quad \int_{\Omega} q^{(0)} d x=0
$$

is recovered. Due to the second order accuracy of the stencils in (34), the equations for $q_{\text {odd }}^{(1)}$ and $q_{\text {odd }}^{(3)}$ are homogeneous so that $q_{\text {odd }}^{(1)}=q_{\text {odd }}^{(3)}=0$ because of unique solvability. In second order, we find $q_{\text {odd }}^{(2)}$ as solution of

$$
\begin{gathered}
\Delta q^{(2)}=\frac{1}{6}\left(\frac{\partial^{3} a_{1}}{\partial x_{1}^{3}}+\frac{\partial^{3} a_{2}}{\partial x_{2}^{3}}\right)-\frac{1}{3}\left(\frac{\partial^{4}}{\partial x_{1}^{4}}+\frac{\partial^{4}}{\partial x_{2}^{4}}\right) q^{(0)} \\
\frac{\partial q^{(2)}}{\partial x_{2}}=-\frac{1}{6} \frac{\partial^{3} q^{(0)}}{\partial x_{2}^{3}} \text { on } \partial \Omega, \quad \int_{\Omega} q^{(2)} d x=0
\end{gathered}
$$

Altogether, we obtain the expansion

$$
q_{\text {odd }}=q_{\text {odd }}^{(0)}+h^{2} q_{\text {odd }}^{(2)}+\mathcal{O}\left(h^{4}\right)
$$


In the next step we can solve the problem on the even sub-grid which uses $q_{\text {odd }}$ in the boundary conditions

$$
\begin{gathered}
\left(L q_{\text {even }}-D \mathbf{a}\right)_{i j}=0 \quad j=0,2, \ldots, N \\
\frac{1}{3}\left[\left(D_{2} q_{\text {even }}-a_{2}\right)_{i,-1}+3\left(D_{2} q_{\text {even }}-a_{2}\right)_{i, 1}\right] \\
=\frac{1}{3}\left(D_{2} q_{\text {odd }}-a_{2}\right)_{i, 2}+\frac{h}{3}\left(D_{1} D_{1} q_{\text {even }}-D_{1} a_{1}\right)_{i, 0}
\end{gathered}
$$

(a corresponding condition applies on the upper boundary). The coefficients in

$$
q_{\mathrm{even}}=q_{\mathrm{even}}^{(0)}+h q_{\mathrm{even}}^{(1)}+h^{2} q_{\mathrm{even}}^{(2)}+h^{3} q_{\mathrm{even}}^{(3)}+\mathcal{O}\left(h^{4}\right) .
$$

are determined with the same procedure as above. It turns out that $q_{\mathrm{even}}^{(0)}=q_{\mathrm{odd}}^{(0)}$ is the solution of $(36)$ and $q_{\text {even }}^{(1)}$ solves

$$
\Delta q^{(1)}=0, \quad \frac{\partial q^{(1)}}{\partial x_{2}}=\frac{1}{4}\left(\frac{\partial^{2} q^{(0)}}{\partial x_{1}^{2}}-\frac{\partial a_{1}}{\partial x_{1}}\right) \quad \text { on } \partial \Omega, \quad \int_{\Omega} q^{(1)} d x=0
$$

In general, the solution to this problem is non-trivial. However, if $\mathbf{a}=\nu \Delta \mathbf{u}$ and $q^{(0)}=p$ are related to a smooth solution of the Stokes equation, a compatibility condition assures that $\partial^{2} q^{(0)} / \partial x_{1}^{2}=\partial a_{1} / \partial x_{1}$ so that, in fact, $q_{\text {even }}^{(1)}=0$ (basically, the Stokes equation implies $\nabla p=\nu \Delta \mathbf{u}$ on the boundary so that the compatibility relation follows by applying an additional $x_{1}$-derivative).

For the coefficient $q_{\text {even }}^{(2)}$, we recover equation $(37)$ with boundary condition

$$
\frac{\partial q_{\mathrm{even}}^{(2)}}{\partial x_{2}}=\frac{\partial q_{\mathrm{odd}}^{(2)}}{\partial x_{2}}
$$

so that also $q_{\text {even }}^{(2)}=q_{\text {odd }}^{(2)}$. Only in third order, we observe a difference between the solutions on the odd and even sub-grids. While $q_{\text {odd }}^{(3)}=0$, the coefficient $q_{\text {even }}^{(3)}$ solves

$$
\begin{gathered}
\Delta q^{(3)}=0, \quad \int_{\Omega} q^{(3)} d x=0, \\
\frac{\partial q^{(3)}}{\partial x_{2}}=\frac{1}{12}\left(\frac{\partial^{4} q^{(0)}}{\partial x_{2}^{4}}-\frac{\partial^{3} a_{2}}{\partial x_{2}^{3}}\right)-\frac{1}{4}\left(\frac{\partial^{2} q^{(2)}}{\partial x_{2}^{2}}+\frac{1}{6} \frac{\partial^{4} q^{(0)}}{\partial x_{2}^{4}}\right) \quad \text { on } \partial \Omega
\end{gathered}
$$

Combining the solutions on the sub-grids we find the expansion

$$
q_{i j}=q_{i j}^{(0)}+h^{2} q_{i j}^{(2)}+h^{3} q_{i j}^{(3)}+(-1)^{j} h^{3} \hat{q}_{i j}^{(3)}+\mathcal{O}\left(h^{4}\right)
$$

where $q^{(0)}, q^{(2)}$ solve $(36)$ and (37) and

$$
q^{(3)}=\frac{1}{2}\left(q_{\text {even }}^{(3)}+q_{\text {odd }}^{(3)}\right), \quad q^{(3)}=\frac{1}{2}\left(q_{\text {even }}^{(3)}-q_{\text {odd }}^{(3)}\right) .
$$


In the case of Stokes (or Navier-Stokes) equation, the Poisson problem is coupled with the evolution equation for the velocity field $\mathbf{u}$, giving rise to a similar expansion

$$
\mathbf{u}_{i j}-\mathbf{u}_{i j}^{(0)}+h^{2} \mathbf{u}_{i j}^{(2)}+h^{3} \mathbf{u}_{i j}^{(3)}+(-1)^{j} h^{3} \hat{\mathbf{u}}_{i j}^{(3)}+\mathcal{O}\left(h^{4}\right) .
$$

The input a to the Poisson problem is given by $\nu \Delta \mathbf{u}$ where, in Chorin's method, the Laplacian is discretized with the usual five-point stencil. Applying this stencil to $(40)$, the term $(-1)^{j} h^{3} \hat{\mathbf{u}}_{i j}^{(3)}$ yields the alternating contribution

$$
(-1)^{j}\left(h\left[\begin{array}{lll} 
& -1 \\
1 & -4 & 1 \\
& -1
\end{array}\right] \hat{\mathbf{u}}^{(3)}\right)_{i j}
$$

Since

$$
h\left[\begin{array}{rrr}
1 & -4 & 1 \\
& -1
\end{array}\right]=-4 h+h^{3}\left(\frac{\partial^{2}}{\partial x_{1}^{2}}-\frac{\partial^{2}}{\partial x_{2}^{2}}\right)+\mathcal{O}\left(h^{5}\right)
$$

the source term a in the Poisson equation contains an alternating, first order contribution so that the problem for $q^{(1)}$ now has a non-trivial alternating source. This implies that the approximate pressure $p$ in the Stokes problem is only first order accurate with an alternating error. (For a more detailed analysis, we refer again to [26].)

Let us now define boundary stencils for the new discretization derived in Section 4 . The discrete gradient $\bar{G}$ is always given by $\bar{D}_{1}, \bar{D}_{2}$ defined in (16). The divergence $\bar{D}$ is also composed of these stencils for points inside the domain $(j=2, \ldots, N-2)$. The corresponding Laplacian has the form

$$
\bar{L}=\bar{D} G=\frac{1}{72}\left[\begin{array}{ccccc}
1 & 4 & 8 & 4 & 1 \\
4 & & -8 & & 4 \\
8 & -8 & -36 & -8 & 8 \\
4 & & -8 & & 4 \\
1 & 4 & 8 & 4 & 1
\end{array}\right]
$$

At points next to the boundary, we modify $\bar{D}_{1}$ and $\bar{D}_{2}$ by deleting boundary weights giving rise to $\bar{D}^{(j)}$ and $\vec{L}^{(j)}=\bar{D}^{(j)} \bar{G}$ for $j=1, N-1$. On the boundary itself, we replace $\bar{D}_{1}$ by zero (as in Chorin's method) and choose half-sided differences for $\bar{D}_{2}$. Although these half-sided stencils do not follow directly from the lattice Boltzmann approach, there is a natural choice based on the observation that $\bar{D}_{2}$ can be written as convex combination of central differences

$$
\bar{D}_{2}=\bar{C} D_{2} \quad \text { with } \quad \bar{C}=\frac{1}{6}\left[\begin{array}{lll}
1 & 4 & 1
\end{array}\right] \text {. }
$$


As in Chorin's method, where $D_{2}$ is replaced by $D_{2}^{(0)}$ (a second order, half-sided approximation with deleted central weight) we set

$$
\bar{D}_{2}^{(0)}=\bar{C} D_{2}^{(0)}=\frac{1}{12 h}\left[\begin{array}{ccc}
-1 & -4 & -1 \\
4 & 16 & 4 \\
& 0 \\
0 & 0
\end{array}\right]
$$

A similar choice at the upper boundary yields $\bar{D}_{2}^{(N)}$ and finally gives rise to $\vec{L}^{(j)}=$ $\bar{D}^{(j)} \bar{G}$ for $j=0, N$.

In contrast to the wide Laplacian (31), the stencil (41) does not decouple odd and even sub-grids. However, the coupling is not very strong which can be seen if $\bar{L}$ is applied to a function which is constant in $x_{1}$ direction. Then, the stencil has the same effect as (31) leading to the decoupling known from Chorin's method. Rewriting the boundary stencils in terms of the interior discretization, we again find two different realizations of the Neumann boundary condition (which is an important structural feature for the occurrence of alternating terms). At the lower boundary, we have

$$
\begin{aligned}
& {\left[\bar{C}\left(\bar{D}_{2} q-a_{2}\right)-\frac{h}{3}\left(\bar{D}_{1} \bar{D}_{1} q-D_{1} a_{1}\right)\right]_{i, 0}=0} \\
& {\left[B\left(\bar{D}_{2} q-a_{2}\right)-\frac{2 h}{3}\left(\bar{D}_{1} \bar{D}_{1} q-D_{1} a_{1}\right)\right]_{i, 0}=0}
\end{aligned}
$$

where $B$ is defined in (33). If the compatibility condition $\partial^{2} q / \partial x_{1}^{2}=\partial a_{1} / \partial x_{1}$ is satisfied, both conditions differ only in third order, exactly as in the case of Chorin's method.

The fundamental difference compared to Chorin's approach is the structure of the Laplacian $\hat{L}(28)$ which is used to discretize the viscosity term $\nu \Delta \mathbf{u}$. We neglect second order terms in the definition of $\hat{L}$ and consider

$$
\hat{L} \mathbf{u}=\left(\begin{array}{c}
\bar{D}_{1}^{2} u_{1}+\tilde{D}_{2}^{2} u_{1} \\
\tilde{D}_{1}^{2} u_{2}+\bar{D}_{2}^{2} u_{2}
\end{array}\right)
$$

Granting that $\mathbf{u}$ has an expansion as in (40), the Laplacian $\hat{L}$ does not amplify the alternating term as the standard five-point stencil. In fact, applying $\hat{L}$ to $h^{3} A \hat{\mathbf{u}}^{(3)}$ (where $A$ is an alternating function with $A_{i j}=(-1)^{j}$ ) leads to an alternating term of the same order

$$
h^{3} \hat{L} A \hat{\mathbf{u}}^{(3)}=h^{3} A\left(\begin{array}{c}
\frac{1}{9} \frac{\partial \hat{u}_{1}^{(3)}}{\partial x_{1}^{2}}+\frac{\partial \hat{u}_{1}^{(3)}}{\partial x_{2}^{2}} \\
\Delta \hat{u}_{2}^{(3)}
\end{array}\right)+\mathcal{O}\left(h^{5}\right) .
$$


In contrast to the five-point Laplacian, the wide stencil $\hat{L}$ requires a special treatment at points next to the boundary. As for the boundary divergence, we consider the participating stencils as convex combinations of usual central differences which are replaced by half-sided approximations if necessary. For example, $\tilde{D}_{2}$ defined in (17) can be written as $\tilde{D}_{2}=\tilde{C} D_{2}$ with $\tilde{C}=\frac{1}{2}\left[\begin{array}{lll}1 & 0 & 1\end{array}\right]$. Close to the boundary, we replace $D_{2}$ by $D_{2, h s}$ defined in (32). Altogether, we set

$$
\left(\hat{L}^{(j)} \mathbf{u}\right)_{i j}=\left(\begin{array}{l}
\tilde{D}_{1} D_{1} u_{1}+\tilde{D}_{2} \tilde{C} D_{2, h s} u_{1} \\
\tilde{D}_{1} D_{1} u_{2}+\bar{D}_{2} \bar{C} D_{2, h s} u_{2}
\end{array}\right)_{i j}, \quad j \in\{1, N-1\}
$$

This modified stencil now again amplifies an alternating term but only by one order

$$
\hat{L}^{(j)} A \hat{\mathbf{u}}^{(3)}=\frac{4}{h} \frac{\partial}{\partial x_{2}} \hat{\mathbf{u}}^{(3)}+\mathcal{O}(1)
$$

Consequently, a second order alternating contribution appears in the calculated pressure and adds to the second order error already present giving rise to a second order accurate scheme. (The amplification can even be avoided altogether by using $D_{2, h s}$ based on twice the grid size. Then, the approximate second $x_{2}$-derivative operates only on the even sub-grid and thus is unaffected by alternating terms.)

Motivated by the analysis of the lattice Boltzmann induced discretization, we can set up other schemes with a similar behavior but with simpler stencils. We just use the feature of a wide Laplacian in the discretization of $\nu \Delta \mathbf{u}$ to avoid amplifications of high order alternating terms. For example, to simplify the numerical effort, one may use, instead of $\hat{L}$, the stencil $\bar{L}$ - with appropriate boundary modifications to approximate the viscous term. Another possibility is to use the wide five point stencil (31). As with the LB-induced discretization, $h^{3} A \hat{\mathbf{u}}^{(3)}$ is not amplified inside the domain and at most by one order next to the boundary so that second order schemes in velocity and pressure are obtained.

\section{Numerical investigations}

Our numerical test problem is taken from [26]. The periodic channel problem (29) is initialized with the velocity field

$$
\begin{aligned}
& u_{1}\left(x_{1}, x_{2}\right)=6 x_{2}\left(1-x_{2}\right)+16\left(2 x_{2}-6 x_{2}^{2}+4 x_{2}^{3}\right) \sin \left(2 \pi x_{1}\right) / 2 \pi \\
& u_{2}\left(x_{1}, x_{2}\right)=-16\left(x_{2}^{2}-2 x_{2}^{3}+x_{2}^{4}\right) \cos \left(2 \pi x_{1}\right)
\end{aligned}
$$

which relaxes to plane Poiseuille flow for $t \rightarrow \infty$. Errors due to time integration are kept small by using very small time steps. The results obtained with Chorin's method and the LB-induced discretization are presented at $t=1$. As mentioned at the end of the previous section, we consider a simplification of the method described above, using the stencil defined by (31) instead of $\hat{L}$. (With $\hat{L}$ instead of $\hat{L}$, the same behavior is observed.) The orders of convergence of the pressure for the different methods are given in the following table: 


\begin{tabular}{|c|c|c|c|}
\hline $\mathrm{N}$ & Chorin & Chorin (filtered) & LB-based \\
\hline 16 & 1.2321 & 0.6655 & 0.2473 \\
\hline 32 & $0.5694(1.11)$ & $0.1604(2.05)$ & $0.0650(1.93)$ \\
\hline 64 & $0.2703(1.07)$ & $0.0359(2.16)$ & $0.0158(2.04)$ \\
\hline 128 & $0.1311(1.04)$ & $0.0065(2.47)$ & $0.0031(2.34)$ \\
\hline
\end{tabular}

Table 1: Absolute pressure errors $\left(* 10^{-3}\right)$ (and estimated convergence rates)

First order convergence for the pressure is found with Chorin's method in contrast to the second order convergence with the lattice Boltzmann based method. This is in accordance with the analytical considerations, since wide Laplacians are used to discretize the viscous terms in the velocity equation. We mention that second order convergence for the pressure can also be obtained with Chorin's method if filtering of the pressure is used, see [26]. However, filtering introduces additional errors so that Chorin's scheme including filtering shows a larger error than the LB type method.

The convergence of velocity is second order in both cases, as shown in table 2 .

\begin{tabular}{|c|c|c|}
\hline $\mathrm{N}$ & \multicolumn{1}{|c|}{ Chorin } & LB-based \\
\hline 16 & 0.8561 & 3.6406 \\
\hline 32 & $0.2141(2.00)$ & $0.9249(1.98)$ \\
\hline 64 & $0.0582(1.88)$ & $0.2215(2.06)$ \\
\hline 128 & $0.0125(2.21)$ & $0.0444(2.32)$ \\
\hline
\end{tabular}

Table 2. Absolute velocity errors $\left(* 10^{-3}\right)$ (and estimated convergence rates)

Here, the absolute errors of the LB method exceed those of Chorin's method by a factor four. This loss of resolution is due to the fact that a wide stencil of size $2 h$. is used to approximate the viscous term instead of a usual five-point stencil as in Chorin's method.

\section{Conclusions}

Starting from a Lattice Boltzmann type discrete velocity model with the diffusion scaling, a relaxation system for an equivalent set of velocity moments is derived. A simple upwind discretization of the kinetic equalion, similar to the one used in the original Lattice Boltzmann scheme, gives rise to a spatial discretization of the moment system. A semi-implicit time discretization which respects the stiffness of the problem then leads to a Chorin-type projection method for the incompressible Navier-Stokes equations as limiting system. In contrast to Chorin's original method, second order convergence of the pressure is observed. This improvement is related to the use of wide stencils in the velocity equation. On the other hand, wide stencils 
reduce resolution so that absolute errors in velocity are larger compared to Chorin's method although both schemes are second order accurate in velocity. The analytical results are based on an analysis of alternating error terms and are supported by numerical investigations.

\section{References}

[1] T. Abe. Derivation of the Lattice Boltzmann Method by means of the discrete ordinate method for the Boltzmann equation. J. Comp. Phys, 131:241-246, 1997.

[2] C. Bardos, F. Golse, and D. Levermore. Fluid dynamic limits of kinetic equations: Formal derivations. J. Stat. Phys., 63:323, 1991.

[3] S. Benzi, R.and Succi and M. Vergassola. The Lattice-Boltzmann equation: Theory and applications. Physics Reports, 222:145-197, 1992.

[4] R.E. Caflisch, S. Jin, and G. Russo. Uniformly accurate schemes for hyperbolic systems with relaxation. SIAM J. Num. Anal., 34:246-281, 1997.

[5] N. Cao, S. Chen, S. Jin, and D. Martinez. Physical symmetry and lattice symmetry in Lattice Boltzmann methods. Phys. Rev. E, 55:21, 1997.

[6] H. Chen, S. Chen, and W. Matthaeus. Recovery of the Navier-Stokes equations using a Lattice-gas Boltzmann method. Physical Review A, 45:5339-5342, 1992.

[7] A.J. Chorin. Numerical solution of the Navier-Stokes equations. Math. Comp., 22:745-762, 1968 .

[8] A. De Masi, R. Esposito, and J.L. Lebowitz. Incompressible Navier Stokes and Euler limits of the Boltzmann equation. CPAM, 42:1189, 1989.

[9] D. d'Humières. Generalized Lattice-Boltzmann Equations in: AIAA Rarefied Gas Dynamics: Theory and Applications. Progress in Astronautics and Aeoronautics, 159:450-458, 1992.

[10] U. Frisch, D. d'Humieres, B. Hasslacher, P. Lallemand, Y. Pomeau, and J.P. Rivet. Lattice gas hydrodynamics in two and three dimensions. Complex Systems, 1:649-707, 1987 .

[11] S. Hou, Q. Zou, S. Chen, G. Doolen, and A.C. Cogley. Simulation of cavity flow by the lattice Boltzmann method. Journal of Computational Physics, 118:329, 1995.

[12] S. Jin and D. Levermore. Numerical schemes for hyperbolic conservation laws with stiff relaxation terms. J. Comp. Phys., 126:449, 1996. 
[13] S. Jin, L. Pareschi, and G. Toscani. Diffusive relaxation schemes for discretevelocity kinetic equations. SIAM I. Num. Anal., 35:2405-2439, 1998.

[14] S. Jin and Z. Xin. The relaxation schemes for systems of conservation laws in arbitrary space dimensions. Comm. Pure Appl. Math., 48:235-276, 1995.

[15] M. Junk. A Finite Difference Interpretation of the Lattice Boltzmann Method. preprint, Universität Kaiserslautern, 1999.

[16] M. Junk. Kinetic schemes in the case of low Mach numbers. J. Comp. Phys, 151:1-22, 1999.

[17] A. Klar. A numerical method for kinetic semiconductor equations in the drift diffusion limit. SIAM J. Sci. Comp., to appear.

[18] A. Klar. An asymptotic-induced scheme for nonstationary transport equations in the diffusive limit. SIAM J. Num. Anal., 35:1073 1094, 1998.

[19] A. Klar. Relaxation schemes for a Lattice Boltzmann type discrete velocity model and numerical Navier Stokes limit. J. Comp. Phys., 148:1-17, 1999.

[20] E.W. Larsen and J.E. Morel. Asymptotic solution of numerical transport problems in optically thick, diffusive regimes II. J. Comp. Phys., 83:212-236, 1989.

[21] T. Platkowski and R. Illner. Discrete velocity models of the Boltzmann equation. SIAM Review, 30:213-255, 1988.

[22] Y.H. Qian, D. d'Humieres, and P. Lallemand. Lattice BGK models for the Navier Stokes equation. Europhys. Letters, 17:479-484, 1992.

[23] M. Reider and J. Sterling. Accuracy of discrete velocity BGK models for the simulation of the incompressible Navier Stokes equations. Computers and Flu$i d s, 24: 459-467,1995$.

[24] Y. Sone. Asymtotic theory of a steady flow of a rarefied gas past bodies for small knudsen numbers. In R. Gatignol and Soubbaramayer, editors, Advances in Kinetic Theory and Continuum Mechanics, Proceedings of a Symposium Held in Honour of Henri Cabannes (Paris), Springer, pages 19-31, 1990.

[25] J. Sterling and S. Chen. Stability analysis of Lattice Boltzmann Methods. $J$. Comp. Phys., 123:196-206, 1996.

[26] B. Wetton. Analysis of the spatial error for a class of finite difference methods for viscous incompressible flow. SIAM J. Num. Anal., 34:723-755, 1997.

[27] B. Wetton. Error analysis for Chorin's original fully discrete projection method and regularizations in space and time. SIAM J. Num. Anal., 34:1683-1697, 1997. 